\title{
Discrete-time prey-predator model with $\theta$-logistic growth for prey incorporating square root functional response
}

\section{P. K. Santra}

To cite this article:

P. K. Santra,"Discrete-time prey-predator model with $\theta$-logistic growth for prey incorporating square root

functional response", Jambura J. Biomath, vol. 1, no. 2, pp. 41-48, 2020

DOI: https://doi.org/10.34312/jjbm.v1i2.7660

(C) 2020 Author(s).

Articles You may be interested in

A stage-structure Rosenzweig-MacArthur model with effect of prey refuge

L. K. Beay and M. Saija

http://dx.doi.org/10.34312/jjbm.v1i1.6891

Analisis kestabilan model predator-prey dengan infeksi penyakit pada prey dan pemanenan proporsional pada predator

S. Maisaroh, Resmawan, and E. Rahmi

http://dx.doi.org/10.34312/jjbm.v1i1.5948

Bifurkasi Hopf pada model Lotka-Volterra orde-fraksional dengan Efek Allee aditif pada predator

H. S. Panigoro and D. Savitri

http://dx.doi.org/10.34312/jjbm.v1i1.6908

Parameters estimation of generalized Richards model for COVID-19 cases in Indonesia using genetic algorithm

M. Rayungsari, M. Aufin, and N. Imamah

http://dx.doi.org/10.34312/jjbm.v1i1.6910

Revitalisasi danau Limboto dengan pengerukan endapan di danau: pemodelan, analisis, dan simulasinya

S. L. Mahmud, N. Achmad, and H. S. Panigoro

http://dx.doi.org/10.34312/jjbm.v1i1.6945 


\title{
Discrete-time prey-predator model with $\theta$-logistic growth for prey incorporating square root functional response
}

\author{
P. K. Santra \\ Abada Nsup School, Abada, Howrah-711313, India \\ Corresponding author.Email: prasunsantra5@gmail.com
}

\begin{abstract}
This article presents the dynamics of a discrete-time prey-predator system with square root functional response incorporating $\theta$-logistic growth. This type of functional response is used to study the dynamics of the prey-predator system where the prey population exhibits herd behavior, i.e., the interaction between prey and predator occurs along the boundary of the population. The existence and stability of fixed points and Neimark-Sacker Bifurcation (NSB) are analyzed. The phase portraits, bifurcation diagrams, and Lyapunov exponents are presented and analyzed for different parameters of the model. Numerical simulations show that the discrete model exhibits rich dynamics as the effect of $\theta$-logistic growth.
\end{abstract}

Keywords: Discrete Prey-Predator Model; $\theta$-Logistic Growth; Lyapunov Exponents; Stability; Neimark-Sacker Bifurcation

\section{Introduction}

For the purpose of system modeling and analysis in biomathematical literature, discrete-time models are more considered an essential tool. Firstly, discrete-time models are more suitable for describing systems which evolve over time. Secondly, compared with continuous-time models, the advantage they offer is that they are generally more direct, more convenient, and more accurate to formulate. Thirdly, recent works have shown that for discrete-time models the dynamics can produce a much richer set of patterns than those observed in continuous-time models [1-4]. In the research articles [5-7], the authors studied the various dynamical behaviours of discrete predator-prey systems with refuge. Stability, bifurcation, chaos are studied by authors [8-11] in discrete-time predator-prey.

In ecology, depending on the abundance of the populations, various authors use different types of functional responses in population models. Hence, the choice of functional response is important to make a realistic model. The useful functional responses are the three Holling-type functional responses. But there are some prey populations which exhibit herd behavior, i.e., the interaction between prey and predator occurs along the boundary of the prey population. This type of interaction cannot be explained fully using the Holling-type responses. In reality, a class of prey population exhibits herd behavior so that the capturing rate of prey by a predator will be different from usual models. For example, the capturing rate of zooplankton by a fish in the ocean is greater than the capturing rate of phytoplankton by a fish. In this case, the phytoplankton exhibits herd behavior. To interpret the herd behavior of the prey population, we took into account the square root of the prey population so that the predator interacts with the prey along the outer corridor of the herd of the prey. Even though the logistic model includes more population growth factors, the basic logistic model is still not good enough. As a large population size continues to grow, the individual growth rate should slow down. This new finding is not included in the classical model. In order to fit data better and address the limitations from the classic logistic model, Gilpin and Ayala presented a new version of the logistic model called "theta-logistic model" $[12,13]$. A new term $\theta$ is added to the classic logistic model. The linear density dependence held by the classical logistic model can be altered to curvilinear. It is this $\theta$ that provides the additional generality and flexibility to explain the impact by the change of individual growth rate parameter $r$ with respect to population density $x$.

The rest of the paper is organized as follows: In Section 2, a discrete-time prey-predator model is formulated. Section 3 deals with the local stability analysis of the proposed model. Bifurcation of the proposed system studied in Section 4. Section 5 presents numerical simulations to support the dynamical analysis of the proposed model. Finally, this paper ends with a conclusion in Section 6. 


\section{Mathematical model}

This works studies the modified Lotka-Volterra prey-predator model with square root functional response incorporating $\theta$-logistic growth. The prey-predator dynamics is governed by following system of equation:

$$
\begin{aligned}
& \frac{d x}{d t}=r x\left(1-\left(\frac{x}{k}\right)^{\theta}\right)-c y \sqrt{x}, \\
& \frac{d y}{d t}=d y \sqrt{x}-f y,
\end{aligned}
$$

with initial condition

$$
x(t) \geq 0, y(t) \geq 0,
$$

where $x$ and $y$ denote the density of prey and predator populations respectively at any time $t$. All involved parameters are kept positive and their biological meanings are as follows: $r$ is the intrinsic per capita growth rate of prey population, $\theta$ is a dimensionless parameter influencing the curvature of the relationship between the exponential rate of growth and prey population density, $k$ is the environmental carrying capacity of prey population, $c$ is the maximal per capita consumption rate of predators, $d$ is the efficiency with which predators convert consumed prey into new predators, and $f$ is the per capita death rate of predators.

To derive a discrete-time model from (1), let $\frac{d x}{d t}=\frac{x_{t+h}-x_{t}}{h}$, and $\frac{d y}{d t}=\frac{y_{t+h}-y_{t}}{h}$, where $x_{t}$ and $y_{t}$ are the densities of the prey and predator populations in discrete time $t$. Let $h \rightarrow 1$ and $f=1$, we have the equations for the $(n+1)^{t h}$ generation of the prey and predator populations replacing $t$ by $n$ as follows:

$$
\begin{aligned}
& x_{n+1}=(r+1) x_{n}\left(1-\frac{r}{(r+1)}\left(\frac{x_{n}}{k}\right)^{\theta}\right)-c y_{n} \sqrt{x_{n}} \\
& y_{n+1}=d y_{n} \sqrt{x_{n}} .
\end{aligned}
$$

Let $\frac{r}{k^{\theta}(r+1)}=\frac{1}{b^{\theta}}$ and $(r+1)=a$, we obtain the discrete-time predator-prey system from (3) as follows:

$$
\begin{aligned}
& x_{n+1}=a x_{n}\left(1-\left(\frac{x_{n}}{b}\right)^{\theta}\right)-c y_{n} \sqrt{x_{n}}, \\
& y_{n+1}=d y_{n} \sqrt{x_{n}}
\end{aligned}
$$

where $a, b, c, d$ and $\theta$ are all positive constants. By the biological meaning of the model variables, we only consider the system in the region $\Omega=\{(x, y): x \geq 0, y \geq 0\}$ in the $(x, y)$-plane.

\subsection{Positivity}

Theorem 1. Every solution of system (1) with initial conditions (2) exists in the interval $[0, \infty)$ and $x(t) \geq 0, y(t) \geq 0$ for all $t \geq 0$.

proof. Since the right hand side of system (1) is completely continuous and locally Lipschitzian on $C$, the solution $(x(t), y(t))$ of (1) with initial conditions (2) exists and is unique on $[0, \xi)$, where $0<\xi \leq+\infty$. From system (1) with initial conditions (2), we have

$$
\begin{aligned}
& x(t)=x(0) \exp \left[\int_{0}^{t}\left\{r\left(1-\left(\frac{x(s)}{k}\right)^{\theta}\right)-\frac{c y(s)}{\sqrt{x(s)}}\right\} d s\right] \geq 0, \\
& y(t)=y(0) \exp \left[\int_{0}^{t}\{d \sqrt{x(s)}-f\} d s\right] \geq 0
\end{aligned}
$$

which completes the proof.

\subsection{Boundedness}

The boundedness of a model guarantees its validity. The following theorem ensures uniform boundedness of all solutions of the system (1).

Theorem 2. All solutions of system (1) are uniformly bounded. 
proof. Let $(x(t), y(t))$ be any solution of system (1). Since $\frac{d x}{d t} \leq r x\left(1-\left(\frac{x}{k}\right)^{\theta}\right)$, we have $\lim _{t \rightarrow \infty} \sup x(t) \leq k$. Let

$$
w=x+\frac{c}{d} y
$$

Then

$$
\frac{d w}{d t}=\frac{d x}{d t}+\frac{c}{d} \frac{d y}{d t}=r x\left(1-\left(\frac{x}{k}\right)^{\theta}\right)-\frac{c f}{d} y \leq 2 r k-\rho w, \text { where } \rho=\min \{r, f\}
$$

Therefore

$$
\frac{d w}{d t}+\rho w \leq 2 r k
$$

Applying a theorem on differential inequalities, we obtain

$$
0 \leq w(x, y) \leq \frac{2 r k}{\rho}+w(x(0), y(0)) \exp (-\rho t)
$$

and for $t \rightarrow \infty$,

$$
0 \leq w(x, y) \leq \frac{2 r k}{\rho}
$$

Thus, all solutions of (1) enter into the region

$$
B=\left\{(x, y): 0 \leq w(x, y) \leq \frac{2 r k}{\rho}+\varepsilon, \text { for any } \varepsilon>0\right\} .
$$

\section{Fixed points and stability analysis}

Fixed points of the system (4) are determined by solving the following non-linear system of equations:

$$
\begin{aligned}
& x=a x\left(1-\left(\frac{x}{b}\right)^{\theta}\right)-c y \sqrt{x} \\
& y=d y \sqrt{x}
\end{aligned}
$$

We get three non-negative fixed points by solving above equations: $(i) P_{0}=(0,0),($ ii $) P_{1}=\left(b\left(\frac{a-1}{a}\right)^{\frac{1}{\theta}}, 0\right)$, $a>1$, and (iii) $P_{2}=\left(x_{2}, y_{2}\right)$, here $x_{2}=\frac{1}{d^{2}}$ and $y_{2}=\frac{1}{c d}\left[a\left(1-\left(\frac{1}{b d^{2}}\right)^{\theta}\right)-1\right], b\left(\frac{a-1}{a}\right)^{\frac{1}{\theta}}>\frac{1}{d^{2}}$.

\subsection{Dynamic behavior of the discrete system}

The discussion about the dynamical behaviour of model (4) is carried out in this sub-section. The Jacobian Matrix $J$ for the system (4) is

$$
J=\left[\begin{array}{cc}
a\left(1-(\theta+1)\left(\frac{x_{n}}{b}\right)^{\theta}\right)-\frac{c y_{n}}{2 \sqrt{x}} & -c \sqrt{x_{n}} \\
\frac{d y_{n}}{2 \sqrt{x_{n}}} & d \sqrt{x_{n}}
\end{array}\right] .
$$

The characteristic equation of the matrix $J$ is $\lambda^{2}-£ \lambda+\Im=0$, where

$$
\begin{aligned}
& £=a\left(1-(\theta+1)\left(\frac{x_{n}}{b}\right)^{\theta}\right)-\frac{c y_{n}}{2 \sqrt{x_{n}}}+d \sqrt{x_{n}}, \\
& \Im=a d \sqrt{x_{n}}\left(1-(\theta+1)\left(\frac{x_{n}}{b}\right)^{\theta}\right) .
\end{aligned}
$$

Hence the discrete-time system (4) is said to be:

(i) a dissipative dynamical system if $\left|a d \sqrt{x_{n}}\left(1-(\theta+1)\left(\frac{x_{n}}{b}\right)^{\theta}\right)\right|<1$,

(ii) a conservative dynamical system if and only if $\left|a d \sqrt{x_{n}}\left(1-(\theta+1)\left(\frac{x_{n}}{b}\right)^{\theta}\right)\right|=1$, and

(iii) an undissipated dynamical system otherwise. 


\subsection{Stability and dynamic behavior at $P_{1}$}

The Jacobian matrix at the fixed point $P_{1}=\left(b\left(\frac{a-1}{a}\right)^{\frac{1}{\theta}}, 0\right)$ is

$$
J=\left[\begin{array}{cc}
1-\theta(a-1) & -c \sqrt{b}\left(\frac{a-1}{a}\right)^{\frac{1}{2 \theta}} \\
0 & d \sqrt{b}\left(\frac{a-1}{a}\right)^{\frac{1}{2 \theta}}
\end{array}\right] .
$$

The equilibrium point $P_{1}$ is said to be:

(i) Sink if $|1-\theta(a-1)|<1$, and $\left|d \sqrt{b}\left(\frac{a-1}{a}\right)^{\frac{1}{2 \theta}}\right|<1$,

(ii) Source if $|1-\theta(a-1)|>1$, and $\left|d \sqrt{b}\left(\frac{a-1}{a}\right)^{\frac{1}{2 \theta}}\right|>1$,

(iii) Saddle if $|1-\theta(a-1)|>1$, and $\left|d \sqrt{b}\left(\frac{a-1}{a}\right)^{\frac{1}{2 \theta}}\right|<1$; or $|1-\theta(a-1)|<1$, and $\left|d \sqrt{b}\left(\frac{a-1}{a}\right)^{\frac{1}{2 \theta}}\right|>1$,

(iv) Non-hyperbolic if $|1-\theta(a-1)|=1$ or $\left|d \sqrt{b}\left(\frac{a-1}{a}\right)^{\frac{1}{2 \theta}}\right|=1$.

\subsection{Dynamic behavior around the interior fixed point}

From the Jacobian matrix at the interior fixed point $P_{2}\left(x_{2}, y_{2}\right)$, we get

$$
\begin{aligned}
& 1-£+\Im=1-a\left(1-(\theta+1)\left(\frac{x_{2}}{b}\right)^{\theta}\right)+\frac{c y_{2}}{2 \sqrt{x_{2}}}-d \sqrt{x_{2}}+a d \sqrt{x_{2}}\left(1-(\theta+1)\left(\frac{x_{2}}{b}\right)^{\theta}\right), \\
& 1+£+\Im=1+a\left(1-(\theta+1)\left(\frac{x_{2}}{b}\right)^{\theta}\right)-\frac{c y_{2}}{2 \sqrt{x_{2}}}+d \sqrt{x_{2}}+a d \sqrt{x_{2}}\left(1-(\theta+1)\left(\frac{x_{2}}{b}\right)^{\theta}\right) .
\end{aligned}
$$

If $1-£+\Im>0$, then interior equilibrium point $P_{2}\left(x_{2}, y_{2}\right)$ is: (i) Sink if $1+£+\Im>0$ and $\Im<1$, (ii) Source if $1+£+\Im>0$ and $\Im>1$, (iii) Saddle if $1+£+\Im<0$, (iv) Non-hyperbolic if $1+£+\Im=0$ and $£ \neq 0,2$, or $£^{2}-4 \Im<0$ and $\Im=1$.

If $1-£+\Im>0, £^{2}-4 \Im<0$, and $\Im=1$, then $P_{2}\left(x_{2}, y_{2}\right)$ can undergo NSB.

\section{Neimark-Sacker bifurcation}

In this section, we discuss NSB for the positive fixed point $P_{2}\left(x_{2}, y_{2}\right)$ of system (4) taking $\theta$ as a bifurcation parameter. NSB is equivalent to the Hopf bifurcation in continuous time system. We discuss the NSB of the discrete prey-predator model (4) at $P_{2}\left(x_{2}, y_{2}\right)$ for the parameters lies in the following set $A=\{(a, b, c, d, \theta)$ : $\left.1-£+\Im>0, £^{2}-4 \Im<0, \Im=1\right\}$.

In analyzing the NSB, $\theta$ is used as the bifurcation parameter. Further $\theta^{*}$ is the perturbation of $\theta$ where $\left|\theta^{*}\right| \lll 1$, we consider a perturbation of the model as follows:

$$
\begin{aligned}
& x_{n+1}=a x_{n}\left(1-\left(\frac{x_{n}}{b}\right)^{\theta+\theta^{*}}\right)-c y_{n} \sqrt{x_{n}} \equiv f\left(x_{n}, y_{n}, \theta^{*}\right), \\
& y_{n+1}=d y_{n} \sqrt{x_{n}} \equiv g\left(x_{n}, y_{n}, \theta^{*}\right) .
\end{aligned}
$$

Let $u_{n}=x_{n}-x_{2}, v_{n}=y_{n}-y_{2}$, then equilibrium $P_{2}\left(x_{2}, y_{2}\right)$ is transformed into the origin, and further expanding $f$ and $g$ as a Taylor series at $\left(u_{n}, v_{n}\right)=(0,0)$ to the third order, the model (5) becomes

$$
\begin{aligned}
u_{n+1}= & \alpha_{1} u_{n}+\alpha_{2} v_{n}+\alpha_{11} u_{n}^{2}+\alpha_{12} u_{n} v_{n}+\alpha_{22} v_{n}^{2}+\alpha_{111} u_{n}^{3}+\alpha_{112} u_{n}^{2} v_{n}+ \\
& \alpha_{122} u_{n} v_{n}^{2}+\alpha_{222} v_{n}^{3}+O\left(\left(\left|u_{n}\right|+\left|v_{n}\right|\right)^{4}\right), \\
v_{n+1}= & \beta_{1} u_{n}+\beta_{2} v_{n}+\beta_{11} u_{n}^{2}+\beta_{12} u_{n} v_{n}+\beta_{22} v_{n}^{2}+\beta_{111} u_{n}^{3}+\beta_{112} u_{n}^{2} v_{n}+ \\
& \beta_{122} u_{n} v_{n}^{2}+\beta_{222} v_{n}^{3}+O\left(\left(\left|u_{n}\right|+\left|v_{n}\right|\right)^{4}\right),
\end{aligned}
$$

where $\alpha_{1}=f_{x}\left(x_{2}, y_{2}, 0\right), \alpha_{2}=f_{y}\left(x_{2}, y_{2}, 0\right), \alpha_{11}=f_{x x}\left(x_{2}, y_{2}, 0\right), \alpha_{12}=f_{x y}\left(x_{2}, y_{2}, 0\right), \alpha_{22}=f_{y y}\left(x_{2}, y_{2}, 0\right)$, $\alpha_{111}=f_{x x x}\left(x_{2}, y_{2}, 0\right), \alpha_{112}=f_{x x y}\left(x_{2}, y_{2}, 0\right), \alpha_{122}=f_{x y y}\left(x_{2}, y_{2}, 0\right), \alpha_{222}=f_{y y y}\left(x_{2}, y_{2}, 0\right), \beta_{1}=g_{x}\left(x_{2}, y_{2}, 0\right)$, 

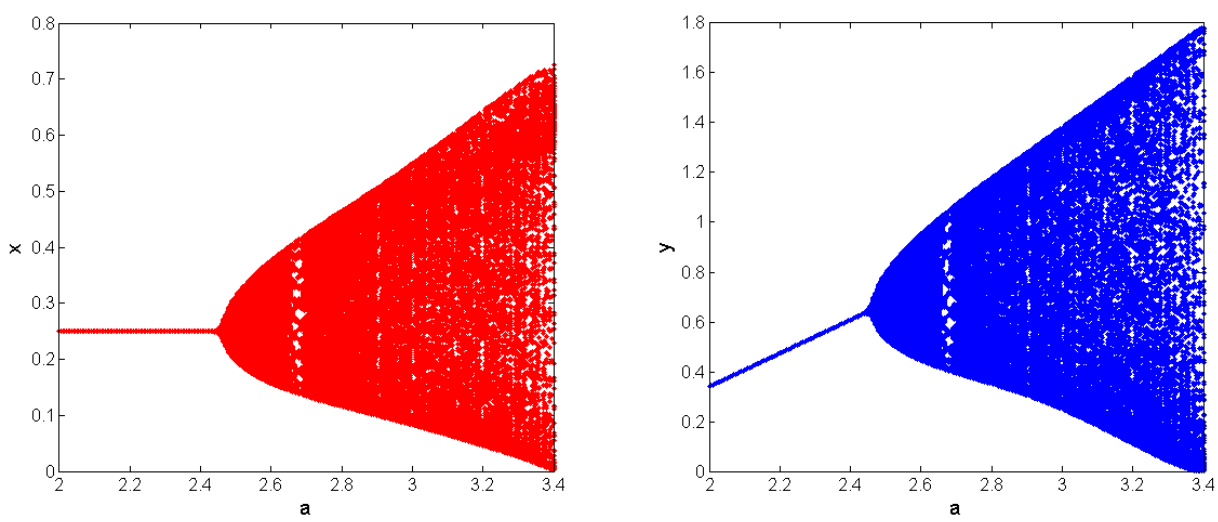

Figure 1. Bifurcation diagram with respect to $a$

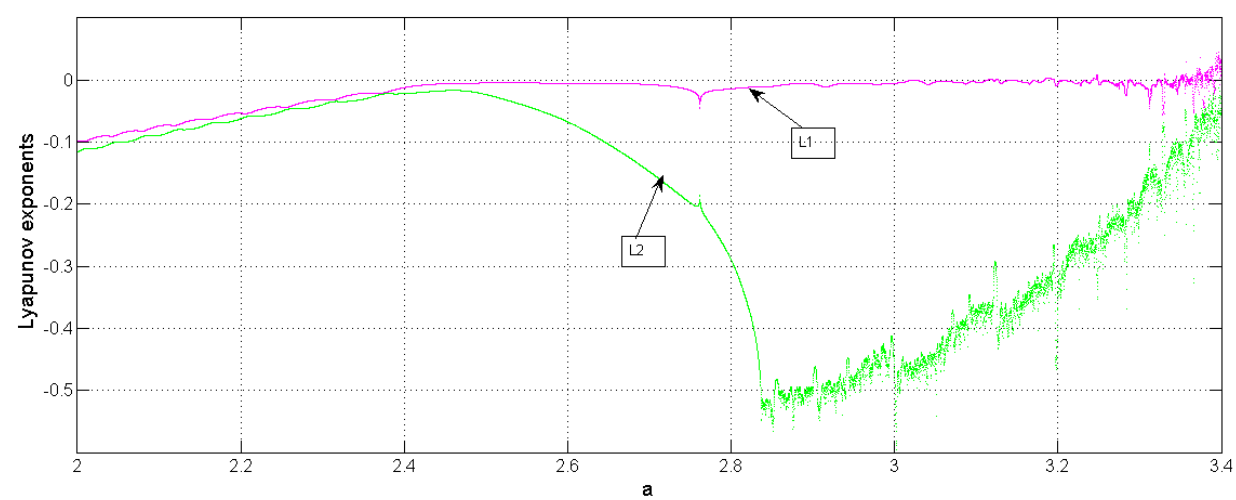

Figure 2. Lyapunov exponents with respect to $a$
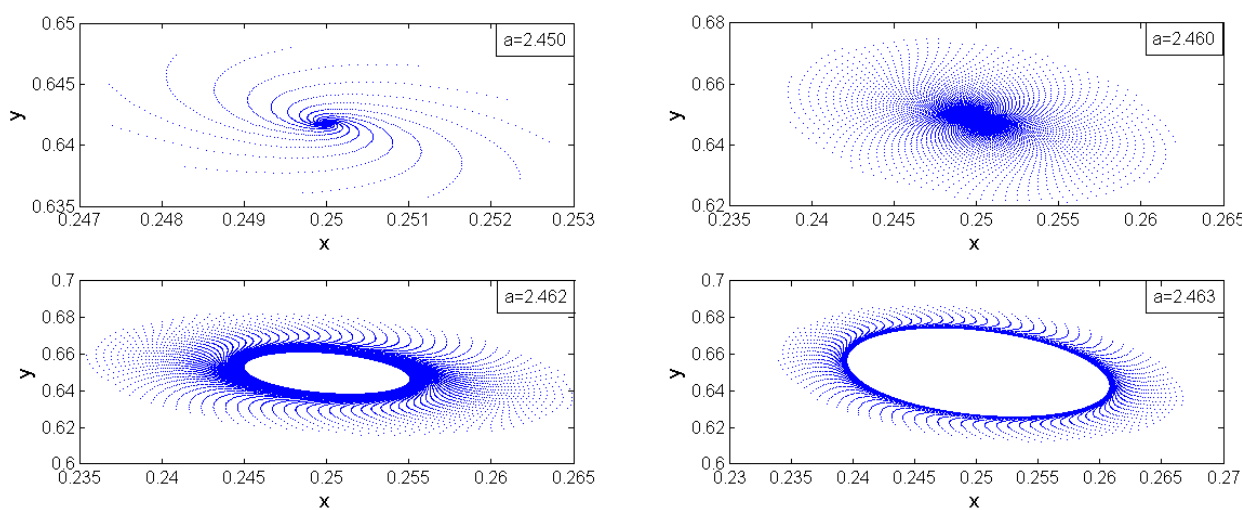

Figure 3. Phase portraits of the system for different values of $a$

$\beta_{2}=g_{y}\left(x_{2}, y_{2}, 0\right), \beta_{11}=g_{x x}\left(x_{2}, y_{2}, 0\right), \beta_{12}=g_{x y}\left(x_{2}, y_{2}, 0\right), \beta_{22}=g_{y y}\left(x_{2}, y_{2}, 0\right), \beta_{111}=g_{x x x}\left(x_{2}, y_{2}, 0\right)$, $\beta_{112}=g_{x x y}\left(x_{2}, y_{2}, 0\right), \beta_{122}=g_{x y y}\left(x_{2}, y_{2}, 0\right)$, and $\beta_{222}=g_{y y y}\left(x_{2}, y_{2}, 0\right)$.

Note that the characteristic equation associated with the linearization of the model (6) at $\left(u_{n}, v_{n}\right)=(0,0)$ is given by $\lambda^{2}-\wp\left(\theta^{*}\right) \lambda+\aleph\left(\theta^{*}\right)=0$. The roots of the characteristic equation are $\lambda_{1,2}\left(\theta^{*}\right)=\frac{\wp\left(\theta^{*}\right) \pm i \sqrt{4 \aleph\left(\theta^{*}\right)-\left(\wp\left(\theta^{*}\right)\right)^{2}}}{2}$.

From $\left|\lambda_{1,2}\left(\theta^{*}\right)\right|=1$, and $\theta^{*}=0$, we have $\left|\lambda_{1,2}\left(\theta^{*}\right)\right|=\left[\aleph\left(\theta^{*}\right)\right]^{\frac{1}{2}}$ and $l=\left[\frac{d\left|\lambda_{1,2}\left(\theta^{*}\right)\right|}{d \theta^{*}}\right]_{h^{*}=0} \neq 0$.

In addition, it is required that when $\theta^{*}=0, \lambda_{1,2}^{i} \neq 1, i=1,2,3,4$, which is equivalent to $\wp(0) \neq-2,-1,1,2$.

To study the normal form, let $\gamma=\operatorname{Im}\left(\lambda_{1,2}\right)$ and $\delta=\operatorname{Re}\left(\lambda_{1,2}\right)$. We define $T=\left[\begin{array}{ll}0 & 1 \\ \gamma & \delta\end{array}\right]$, and using the 

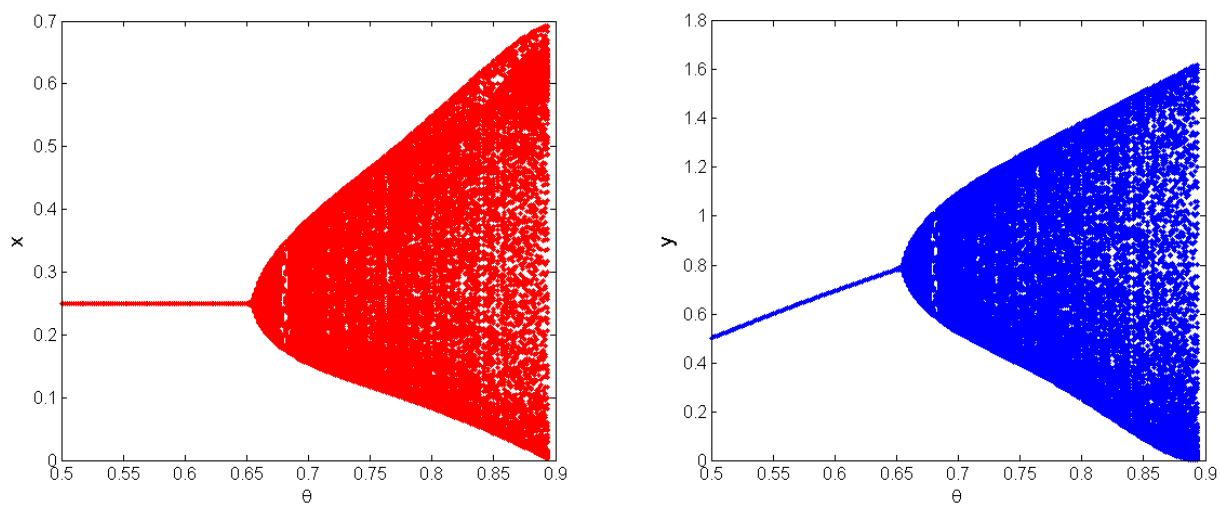

Figure 4. Bifurcation diagram with respect to $\theta$

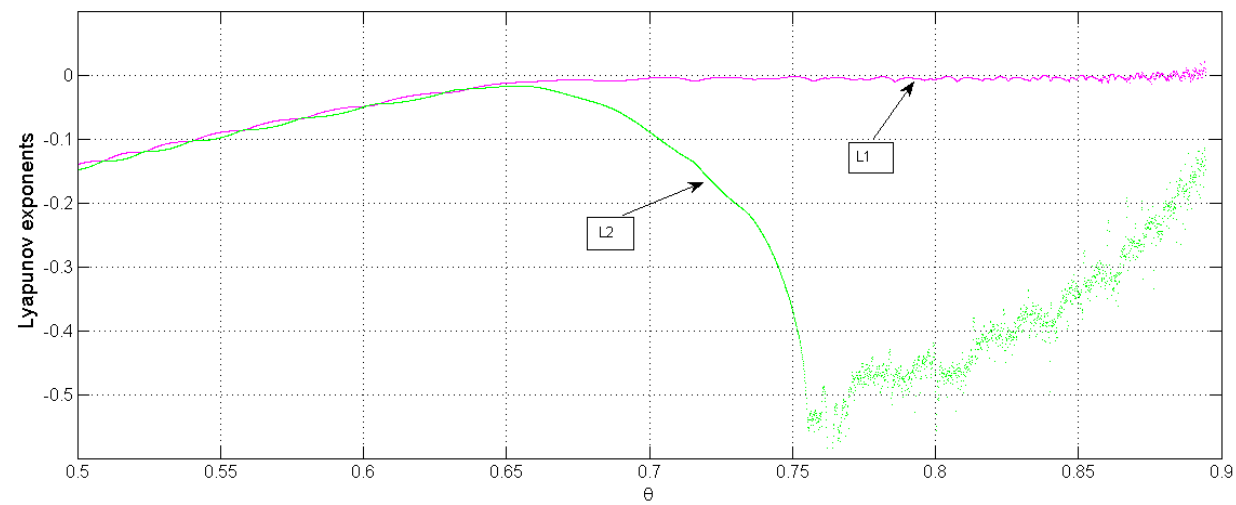

Figure 5. Lyapunov exponents with respect to $\theta$
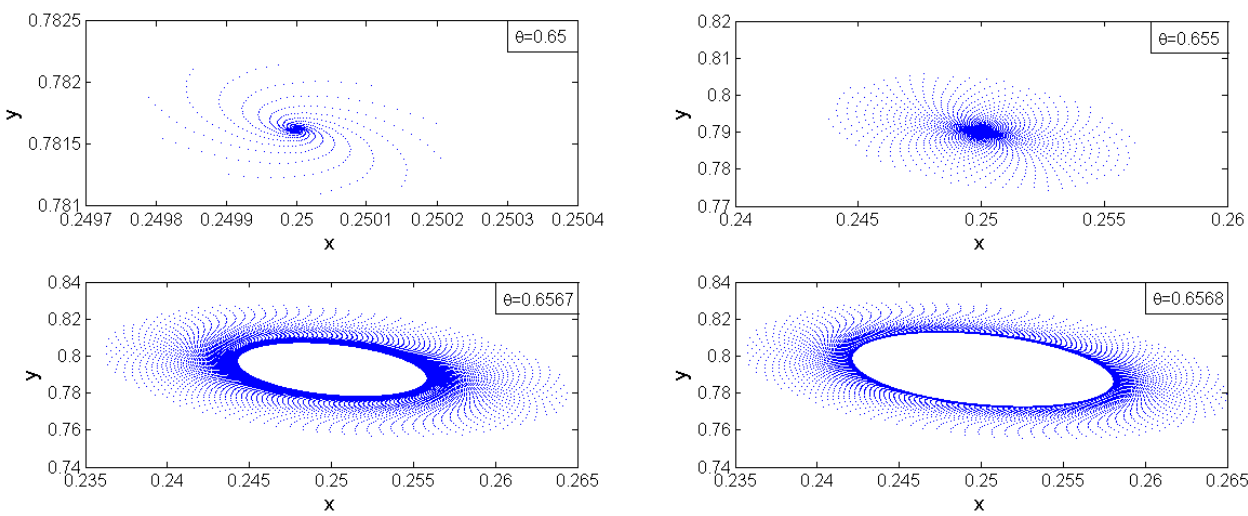

Figure 6. Phase portraits of the system for different values of $a$

transformation $\left[\begin{array}{l}u_{n} \\ v_{n}\end{array}\right]=T\left[\begin{array}{l}\bar{x}_{n} \\ \bar{y}_{n}\end{array}\right]$, the model (6) becomes

$$
\begin{aligned}
& \bar{x}_{n+1}=\delta \bar{x}_{n}-\gamma \bar{y}_{n}+f_{1}\left(\bar{x}_{n}, \bar{y}_{n}\right), \\
& \bar{y}_{n+1}=\gamma \bar{x}_{n}+\delta \bar{y}_{n}+g_{1}\left(\bar{x}_{n}, \bar{y}_{n}\right),
\end{aligned}
$$

where the functions $f_{1}$ and $g_{1}$ denote the terms in the model (7) in variables $\left(\bar{x}_{n}, \bar{y}_{n}\right)$ with the order at least two. In order to undergo NSB, we require that the following discriminatory quantity $\Omega$ be nonzero:

$$
\Omega=-\operatorname{Re}\left[\frac{(1-2 \bar{\lambda}) \bar{\lambda}^{2}}{1-\lambda} \xi_{11} \xi_{20}\right]-\frac{1}{2}\left|\xi_{11}\right|^{2}-\left|\xi_{02}\right|^{2}+\operatorname{Re}\left(\bar{\lambda} \xi_{21}\right),
$$


where

$$
\begin{aligned}
\xi_{20}= & \frac{\delta}{8}\left(2 \beta_{22}-\delta \alpha_{22}-\alpha_{12}+4 \gamma \alpha_{22}+i\left(4 \gamma \alpha_{22}-2 \alpha_{22}-2 \delta \alpha_{22}\right)\right)+\frac{\gamma}{4} \alpha_{12} \\
& +i \frac{1}{8}\left(4 \gamma \beta_{22}+2 \gamma^{2} \alpha_{22}-2 \alpha_{11}\right)+\frac{\beta_{12}}{8}+\frac{\delta \alpha_{11}-2 \beta_{11}+\delta^{3} \alpha_{22}-\delta^{2} \beta_{22}-\delta^{2} \alpha_{12}+\delta \beta_{12}}{4 \gamma}, \\
\xi_{11}= & \frac{\gamma}{2}\left(\beta_{22}-\delta \alpha_{22}\right)+i \frac{1}{2}\left(\gamma^{2} \alpha_{22}+\alpha_{11}+\delta \alpha_{12}+\delta^{2} \alpha_{22}\right)+\frac{\beta_{11}-\delta \alpha_{11}+\delta \beta_{12}-\delta^{2} \alpha_{12}-2 \delta^{2} \beta_{22}+2 \delta^{3} \alpha_{22}}{2 \gamma}, \\
\xi_{02}= & \frac{1}{4} \gamma\left(2 \delta \alpha_{22}+\alpha_{12}+\beta_{22}\right)+i \frac{1}{4}\left(\beta_{12}+2 \delta \beta_{22}-2 \delta \alpha_{12}-\alpha_{11}\right)-\frac{\beta_{11}-\delta \alpha_{11}+\delta \beta_{12}-\delta^{2} \alpha_{12}+\delta^{2} \beta_{22}-\delta^{3} \alpha_{22}}{4 \gamma} \\
& +\frac{1}{4} \alpha_{22} i\left(\gamma^{2}-3 \delta^{2}\right), \\
\xi_{21}= & \frac{3}{8} \beta_{222}\left(\gamma^{2}+\delta^{2}\right)+\frac{\beta_{112}}{8}+\frac{\delta}{4} \alpha_{112}+\frac{\delta}{4} \beta_{122}+\alpha_{122}\left(\frac{\gamma^{2}}{8}+\frac{3 \delta^{2}}{8}-\frac{\delta}{4}\right)+\frac{3}{8} \alpha_{111}+i \frac{3}{8} \alpha_{222}\left(\gamma^{2}+2 \delta^{2}\right) \\
& +i \frac{3 \gamma \delta}{8} \alpha_{122}-\frac{1}{8} \beta_{122} \gamma i-i \frac{3 \gamma \delta}{8} \beta_{222}-i \frac{3 \beta_{111}-3 \delta \alpha_{111}}{8 \gamma}-i \frac{3 \delta \beta_{112}-3 \delta^{2} \alpha_{112}}{8 \gamma}-i \frac{3 \delta^{2} \beta_{122}-3 \delta^{3} \alpha_{122}}{8 \gamma} \\
& -i \frac{3 \delta^{3} \beta_{222}-3 \delta^{4} \alpha_{222}}{8 \gamma} .
\end{aligned}
$$

Finally, we have the following result from the above analysis.

Theorem 3. If $\Omega \neq 0$ then the model (4) undergoes $N S B$ at $P_{2}\left(x_{2}, y_{2}\right)$ when the parameter $\theta^{*}$ varies in a small neighborhood of the origin. If $\Omega<0(\Omega>0)$, then an attrcting (repelling) invariant closed curve bifurcates from $P_{2}\left(x_{2}, y_{2}\right)$ for $\theta^{*}>0\left(\theta^{*}<0\right)$.

\section{Numerical simulations}

In this section, some numerical simulations are provided to confirm our analytical and mathematical investigations.

Example 1. Choosing parameters $a \in[2,3.4], b=1.0, c=0.5, d=2.0, \theta=0.8$, and with initial condition $(0.6,0.4)$ system $(4)$ undergoes NSB when $a \approx 2.461001$. Bifurcation diagrams, Lyapunov exponents and and phase portraits of the corresponding system are depicted in Figures 1 to 3 respectively.

Example 2. Choosing parameters $a=3.0, b=1.0, c=0.5, d=2.0, \theta \in[0.5,0.9]$, and with initial condition $(0.6,0.4)$ system $(4)$ undergoes NSB when $\theta \approx 0.655555$. Bifurcation diagrams, Lyapunov exponents and and phase portraits of the corresponding system are depicted in Figures 4 to 6 respectively.

\section{Conclusion}

In this paper, the effects of $\theta$-logistic growth for prey on a prey-predator interaction are studied through the analytical and graphical approach. We have discussed the effects concerning the local stability of the interior equilibrium point and the long-term dynamics of the interacting populations. The results show that the impact of $\theta$-logistic growth for prey can destabilize the interior equilibrium point of the proposed prey-predator model. We have studied the bifurcations of the proposed discrete prey-predator model. It is shown that the model exhibits Neimark-Sacker Bifurcation, as the values of parameters vary.

\section{References}

[1] A. A. Elsadany, "Dynamical complexities in a discrete-time food chain," Computational Ecology and Software, vol. 2, no. 2, pp. 124-139, 2012.

[2] J. Huang, S. Liu, S. Ruan, and D. Xiao, "Bifurcations in a discrete predator-prey model with nonmonotonic functional response," Journal of Mathematical Analysis and Applications, vol. 464, no. 1, pp. 201-230, 2018.

[3] M. Gámez, I. López, C. Rodríguez, Z. Varga, and J. Garay, “Ecological monitoring in a discrete-time prey-predator model," Journal of Theoretical Biology, vol. 429, pp. 52-60, 2017.

[4] M. Zhao and Y. Du, "Stability of a discrete-time predator-prey system with Allee effect," Nonlinear Analysis and Differential Equations, vol. 4, no. 5, pp. 225-233, 2016.

[5] P. K. Santra and G. S. Mahapatra, "Dynamical study of discrete-time prey-predator model with constant prey refuge under imprecise biological parameters," Journal of Biological Systems, vol. 28, no. 03, pp. 681-699, 2020. 
[6] P. K. Santra, G. S. Mahapatra, and G. R. Phaijoo, "Bifurcation and chaos of a discrete predator-prey model with Crowley-Martin functional response incorporating proportional prey refuge," Mathematical Problems in Engineering, vol. 2020, pp. 1-18, 2020.

[7] R. Ma, Y. Bai, and F. Wang, “Dynamical behavior analysis of a two-dimensional discrete predator-prey model with prey refuge and fear factor," Journal of Applied Analysis and Computation, vol. 10, no. 4, pp. 1683-1697, 2020.

[8] A. Singh and P. Deolia, "Dynamical analysis and chaos control in discrete-time prey-predator model," Communications in Nonlinear Science and Numerical Simulation, vol. 90, p. 105313, 2020.

[9] A. Q. Khan and T. Khalique, "Bifurcations and chaos control in a discrete-time biological model," International Journal of Biomathematics, p. 2050022, 2020.

[10] P. Chakraborty, U. Ghosh, and S. Sarkar, "Stability and bifurcation analysis of a discrete prey-predator model with square-root functional response and optimal harvesting," Journal of Biological Systems, vol. 28, no. 01, pp. 91-110, 2020.

[11] G. Blé, M. A. Dela-Rosa, and I. Loreto-Hernández, "Neimark-Sacker bifurcation analysis in an intraguild predation model with general functional responses," Journal of Difference Equations and Applications, vol. 26, no. 2, pp. 223-243, 2020.

[12] M. E. Gilpin and F. J. Ayala, "Global Models of Growth and Competition," Proceedings of the National Academy of Sciences, vol. 70, no. 12, pp. 3590-3593, 1973.

[13] F. Clark, B. W. Brook, S. Delean, H. Reşit Akçakaya, and C. J. A. Bradshaw, "The theta-logistic is unreliable for modelling most census data," Methods in Ecology and Evolution, vol. 1, no. 3, pp. 253-262, 2010. 


\section{Submit your manuscript at http://ejurnal.ung.ac.id/}

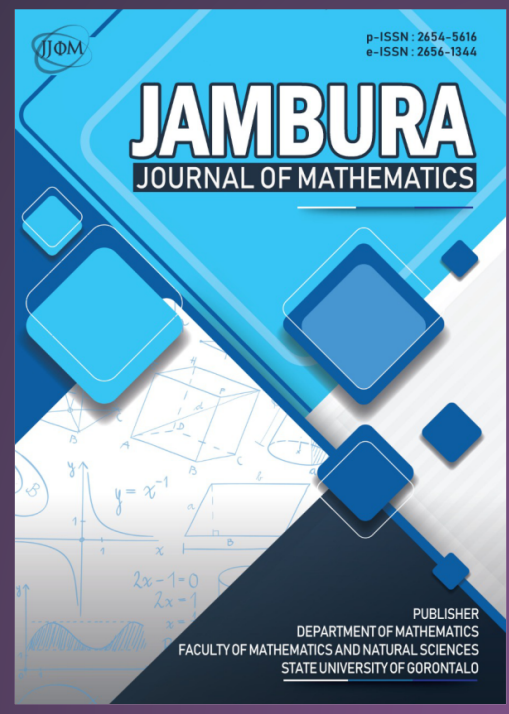

Jambura Journal of Mathematics

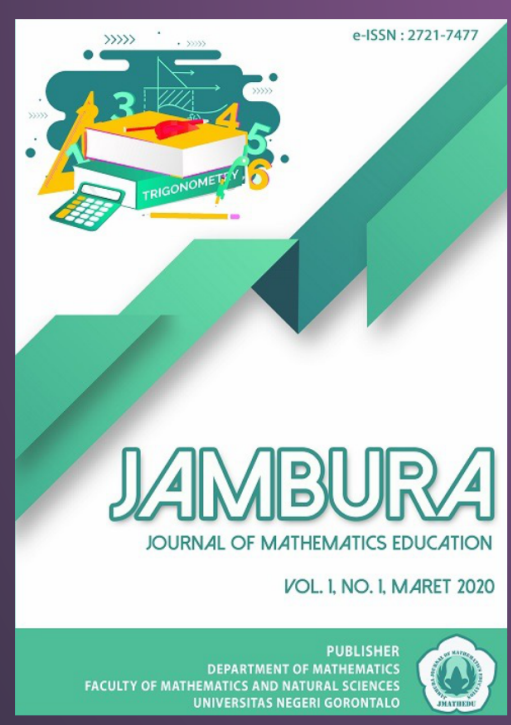

Jambura Journal of Mathematics Education
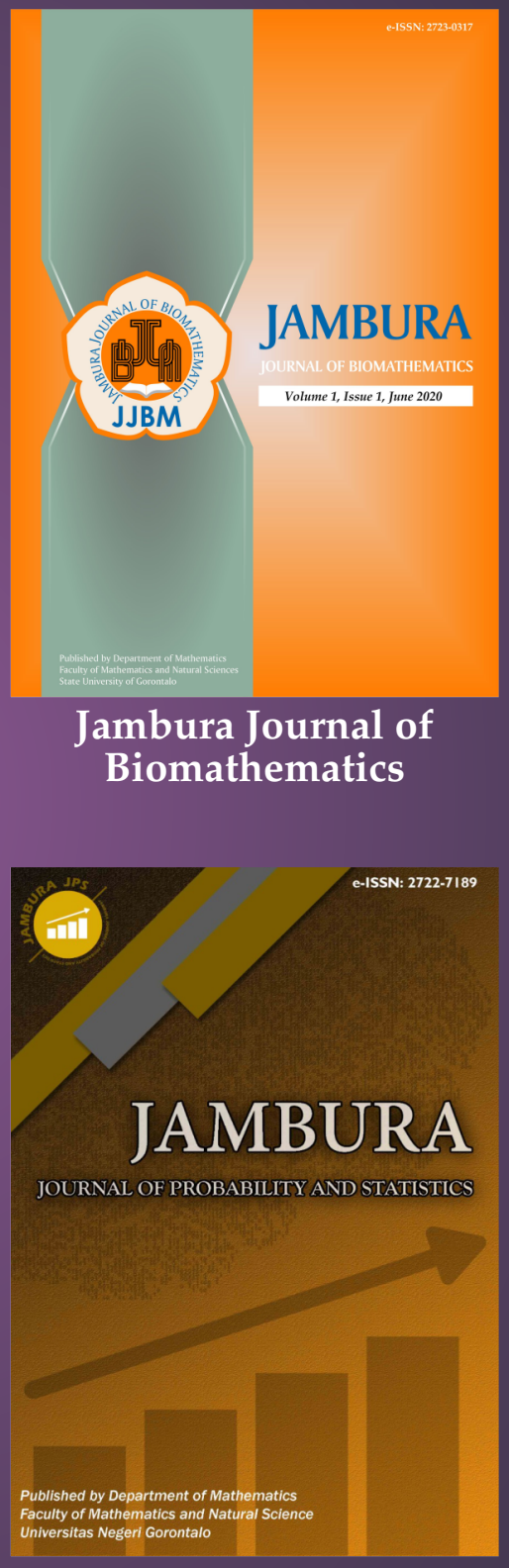

Jambura Journal of Probability and Statistics

Published by

Department of Mathematics

Faculty of Mathematics and Natural Sciences

State University of Gorontalo 